\title{
FIXED POINT THEOREMS IN UNIFORM SPACE ENDOWED WITH GRAPH
}

\author{
MUHAMMAD USMAN ALI, FAHIMUDDIN, TAYYAB KAMRAN, \\ AND ERDAL KARAPINAR
}

Received 02 June, 2016

\begin{abstract}
In this paper, we shall introduce the concepts of $F_{G}$-contraction and $\psi_{G}$-contraction in uniform space endowed with graph to investigate the existence of a fixed point of mappings satisfying these notions. We shall also introduce a common fixed point theorem for pair of mappings satisfying the notion of $\psi_{G}$-contraction in uniform space endowed with graph.
\end{abstract}

2010 Mathematics Subject Classification: 46T99; 47H10; 54H25

Keywords: $F_{G}$-contraction, $\psi_{G}$-contraction, $E_{S}$-distance, uniform space

\section{INTRODUCTION}

Very recently, Wardowski [19] categorized the family of mappings into a new family, denoted by $F$ or $\mathfrak{F}$, to introduce a new contraction condition, the $F$-contraction. As it is expected, it extend the famous Banach contraction mapping principle. Inspired the result of Wardowski, [19], a number of different approaches has been reported, see $[8,13,15-17]$ and related references therein.

On the other hand, Czerwik [9] extended the notion of metric by introducing a new notion, $b$-metric. Indeed, Czerwik [9] modified the triangle inequality that causes to several differences in the related topology, and hence in convergence of a sequence, Hausdorffness of the topology etc. We also mention that Jachymski [10] successively set-up the Banach contraction mapping principle in the frame of a complete metric space endowed with a directed graph, Jachymski [10]. Later, this interesting contribution has been appreciated by several authors $[5,6,11,12,14,18]$.

The purpose of this paper is to introduce and discuss some new fixed point theorems and common fixed point theorems for mappings in uniform space endowed with graph satisfying the $F_{G}$-contraction, and $\psi_{G}$-contraction. Further, we introduce and use the notion of $E_{S}$-distance in this paper.

Some basic definitions and fundamental results are recollected for the sake of completeness. Let $X$ be a nonempty set. A nonempty family, $\vartheta$ of subsets of $X \times X$ is called the uniform structure of $X$, if it satisfies the following properties: 
(i) if $G$ is in $\vartheta$, then $G$ contains the diagonal $\{(x, x) \mid x \in X\}$;

(ii) if $G$ is in $\vartheta$ and $H$ is a subset of $X \times X$ which contains $G$, then $H$ is in $\vartheta$;

(iii) if $G$ and $H$ are in $\vartheta$, then $G \cap H$ is in $\vartheta$;

(iv) if $G$ is in $\vartheta$, then there exists $H$ in $\vartheta$, such that, whenever $(x, y)$ and $(y, z)$ are in $H$, then $(x, z)$ is in $G$;

(v) if $G$ is in $\vartheta$, then $\{(y, x) \mid(x, y) \in G\}$ is also in $\vartheta$.

The pair $(X, \vartheta)$ is called a uniform space and the element of $\vartheta$ is called entourage or neighborhood or surrounding. The pair $(X, \vartheta)$ is called a quasiuniform space (see e.g. [20] ) if property (v) is omitted

Let $\Delta=\{(x, x) \mid x \in X\}$ be the diagonal of a non-empty set $X$. For $V, W \in X \times X$, we shall use the following setting in the sequel

$$
V \circ W=\{(x, y) \mid \text { there exists } z \in X:(x, z) \in W \text { and }(z, y) \in V\}
$$

and

$$
V^{-1}=\{(x, y) \mid(y, x) \in V\} .
$$

For a subset $V \in \vartheta$, a pair of points $x$ and $y$ are said to be $V$-close if $(x, y) \in V$ and $(y, x) \in V$. Moreover, a sequence $\left\{x_{n}\right\}$ in $X$ is called a Cauchy sequence for $\vartheta$, if for any $V \in \vartheta$, there exists $N \geq 1$ such that $x_{n}$ and $x_{m}$ are $V$-close for $n, m \geq N$. For $(X, \vartheta)$, there is a unique topology $\tau(\vartheta)$ on $X$ generated by $V(x)=\{y \in X \mid(x, y) \in$ $V\}$ where $V \in \vartheta$.

A sequence $\left\{x_{n}\right\}$ in $X$ is convergent to $x$ for $\vartheta$, denoted by $\lim _{n \rightarrow \infty} x_{n}=x$, if for any $V \in \vartheta$, there exists $n_{0} \in \mathbb{N}$ such that $x_{n} \in V(x)$ for every $n \geq n_{0}$. A uniform space $(X, \vartheta)$ is called Hausdorff if the intersection of all the $V \in \vartheta$ is equal to $\Delta$ of $X$, that is, if $(x, y) \in V$ for all $V \in \vartheta$ implies $x=y$. If $V=V^{-1}$ then we shall say that a subset $V \in \vartheta$ is symmetrical. Throughout the paper, we shall assume that each $V \in \vartheta$ is symmetrical. For more details, see e.g. [1-4]

Now, we shall recall the notions of $A$-distance and $E$-distance.

Definition 1. [2,3] Let $(X, \vartheta)$ be a uniform space. A function $p: X \times X \longrightarrow[0, \infty)$ is said to be an $A$-distance if for any $V \in \vartheta$ there exists $\delta>0$ such that if $p(z, x) \leq \delta$ and $p(z, y) \leq \delta$ for some $z \in X$, then $(x, y) \in V$.

Definition 2. [2,3] Let $(X, \vartheta)$ be a uniform space. A function $p: X \times X \longrightarrow[0, \infty)$ is said to be an $E$-distance if

(i) $p$ is an $A$-distance,

(ii) $p(x, y) \leq p(x, z)+p(z, y), \forall x, y, z \in X$.

Example 1. [2,3] Let $(X, \vartheta)$ be a uniform space and let $d$ be a metric on $X$. It is evident that $\left(X, \vartheta_{d}\right)$ is a uniform space where $\vartheta_{d}$ is a set of all subsets of $X \times X$ containing a "band" $U_{\epsilon}=\left\{(x, y) \in X^{2} \mid d(x, y)<\epsilon\right\}$ for some $\epsilon>0$. Moreover, if $\vartheta \subseteq \vartheta_{d}$, then $d$ is an $E$-distance on $(X, \vartheta)$. 
Lemma 1. [2,3] Let $(X, \vartheta)$ be a Hausdorff uniform space and $p$ be an A-distance on $X$. Let $\left\{x_{n}\right\}$ and $\left\{y_{n}\right\}$ be sequences in $X$ and $\left\{\alpha_{n}\right\},\left\{\beta_{n}\right\}$ be sequences in $[0, \infty)$ converging to 0 . Then, for $x, y, z \in X$, the following results hold:

(a) If $p\left(x_{n}, y\right) \leq \alpha_{n}$ and $p\left(x_{n}, z\right) \leq \beta_{n}$ for all $n \in \mathbb{N}$, then $y=z$. In particular, if $p(x, y)=0$ and $p(x, z)=0$, then $y=z$.

(b) If $p\left(x_{n}, y_{n}\right) \leq \alpha_{n}$ and $p\left(x_{n}, z\right) \leq \beta_{n}$ for all $n \in \mathbb{N}$, then $\left\{y_{n}\right\}$ converges to $z$.

(c) If $p\left(x_{n}, x_{m}\right) \leq \alpha_{n}$ for all $n, m \in \mathbb{N}$ with $m>n$, then $\left\{x_{n}\right\}$ is a Cauchy sequence in $(X, \vartheta)$.

Let $p$ be an $A$-distance. A sequence in a uniform space $(X, \vartheta)$ with an $A$-distance is said to be a $p$-Cauchy if for every $\epsilon>0$ there exists $n_{0} \in \mathbb{N}$ such that $p\left(x_{n}, x_{m}\right)<\epsilon$ for all $n, m \geq n_{0}$.

Definition 3. [2,3] Let $(X, \vartheta)$ be a uniform space and $p$ be an $A$-distance on $X$.

(i) $X$ is $S$-complete if for every $p$-Cauchy sequence $\left\{x_{n}\right\}$, there exists $x$ in $X$ with $\lim _{n \rightarrow \infty} p\left(x_{n}, x\right)=0$.

(ii) $X$ is $p$-Cauchy complete if for every $p$-Cauchy sequence $\left\{x_{n}\right\}$, there exists $x$ in $X$ with $\lim _{n \rightarrow \infty} x_{n}=x$ with respect to $\tau(\vartheta)$.

(iii) $T: X \rightarrow X$ is $p$-continuous if $\lim _{n \rightarrow \infty} p\left(x_{n}, x\right)=0$ implies $\lim _{n \rightarrow \infty} p\left(T\left(x_{n}\right), T(x)\right)=0$.

Remark 1. Let $(X, \vartheta)$ be a Hausdorff uniform space which is $S$-complete. If a sequence $\left\{x_{n}\right\}$ be a $p$-Cauchy sequence, then we have $\lim _{n \rightarrow \infty} p\left(x_{n}, x\right)=0$. Regarding Lemma $1(\mathrm{~b})$, We derive that $\lim _{n \rightarrow \infty} x_{n}=x$ with respect to the topology $\tau(\vartheta)$ and hence $S$-completeness implies $p$-Cauchy completeness.

Cosentino et al. [7] modify the $\mathfrak{F}$-family introduced by Wardowski [19], in the setting of $b$-metric spaces as follows:

Definition 4. [7] Let $s \geq 1$ be a real number. Denote by $\mathfrak{F}_{s}$ the family of all functions $F:(0, \infty) \rightarrow \mathbb{R}$ satisfying the following conditions:

$\left(F_{1}\right) F$ is strictly increasing, that is, for each $a_{1}, a_{2} \in(0, \infty)$ with $a_{1}<a_{2}$, we have $F\left(a_{1}\right)<F\left(a_{2}\right)$;

$\left(F_{2}\right)$ for each sequence $\left\{\mathfrak{d}_{n}\right\}$ of positive real numbers we have $\lim _{n \rightarrow \infty} \mathfrak{d}_{n}=0$ if and only if $\lim _{n \rightarrow \infty} F\left(\mathfrak{d}_{n}\right)=-\infty$;

$\left(F_{3}\right)$ for each sequence $\left\{\mathfrak{d}_{n}\right\}$ of positive real numbers with $\lim _{n \rightarrow \infty} \mathfrak{d}_{n}=0$, there exists $k \in(0,1)$ such that $\lim _{n \rightarrow \infty} \mathfrak{d}_{\mathfrak{n}}{ }^{k} F\left(\mathfrak{d}_{\mathfrak{n}}\right)=0$.

$\left(F_{4}\right)$ for each sequence $\left\{\mathfrak{d}_{n}\right\}$ of positive real numbers such that $\tau+F\left(s \mathfrak{d}_{n}\right) \leq$ $F\left(\mathfrak{d}_{n-1}\right)$ for each $n \in \mathbb{N}$ and some $\tau>0$, then $\tau+F\left(s^{n} \mathfrak{d}_{n}\right) \leq F\left(s^{n-1} \mathfrak{d}_{n-1}\right)$ for each $n \in \mathbb{N}$.

Cosentino et al. [7] also showed that the following functions belong to $\mathfrak{F}_{s}$.

- $F(x)=x+\ln x$, for each $x>0$.

- $F(x)=\ln x$, for each $x>0$. 


\section{MAIN RESULTS}

We will start this section with the following definition:

Definition 5. Let $(X, \vartheta)$ be a uniform space. A function $p: X \times X \longrightarrow[0, \infty)$ is said to be an $E_{S}$-distance if

(i) $p$ is an $A$-distance,

(ii) $p(x, y) \leq s[p(x, z)+p(z, y)], \forall x, y, z \in X$ and for some $s \geq 1$.

Example 2. Let $(X, \vartheta)$ be a uniform space and let $d$ be a $b$-metric on $X$. Then clearly, $\left(X, \vartheta_{d}\right)$ is a uniform space where $\vartheta_{d}$ is a set of all subsets of $X \times X$ containing a "band" $U_{\epsilon}=\left\{(x, y) \in X^{2} \mid d(x, y)<\epsilon\right\}$ for some $\epsilon>0$. Moreover, if $\vartheta \subseteq \vartheta_{d}$, then $d$ is an $E_{S}$-distance on $(X, \vartheta)$.

Throughout this section, we assume that $G=(V, E)$ is a directed graph such that the set of its vertices $V$ coincides with $X$ (i.e., $V=X$ ) and the set of its edges $E$ is such that $E \supseteq \triangle$, where $\triangle=\{(x, x): x \in X\}$. Further, assume that $G$ has no parallel edges. A mapping $T: X \rightarrow X$ is $p_{G}$-continuous if for each sequence $\left\{x_{n}\right\} \subseteq X$ with $\left(x_{n}, x_{n+1}\right) \in E$ for all $n \in \mathbb{N}$ and $\lim _{n \rightarrow \infty} p\left(x_{n}, x\right)=0$, then we have $\lim _{n \rightarrow \infty} p\left(T x_{n}, T x\right)=0$.

Definition 6. Let $(X, \vartheta)$ be a uniform space endowed with the graph $G$ and $p$ is an $E_{s}$-distance on $X$. A mapping $T: X \rightarrow X$ is a $F_{G}$-contraction, if there exist $F \in \mathfrak{F}_{s}$ and $\tau>0$, such that, for each $(x, y) \in E$, we have

$$
\tau+F(\operatorname{sp}(T x, T y)) \leq F(p(x, y)),
$$

whenever $\min \{p(T x, T y), p(x, y)\}>0$.

Theorem 1. Let $(X, \vartheta)$ be a $S$-complete Hausdorff uniform space endowed with the graph $G$ and $p$ is an $E_{S}$-distance on $X$. Let $T: X \rightarrow X$ is an $F_{G}$-contraction satisfying the following conditions:

(i) $T$ is edge preserving, that is, for $(x, y) \in E$, we have $(T x, T y) \in E$;

(ii) there exists $x_{0} \in X$ such that $\left(x_{0}, T x_{0}\right) \in E$ and $\left(T x_{0}, x_{0}\right) \in E$;

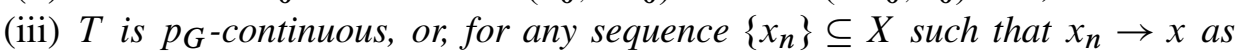
$n \rightarrow \infty$ and $\left(x_{n}, x_{n+1}\right) \in E$ for each $n \in \mathbb{N}$, we have $\left(x_{n}, x\right) \in E$ for each $n \in \mathbb{N}$.

Then $T$ has a fixed point.

Proof. By hypothesis (ii), there exists $x_{0} \in X$ such that $\left(x_{0}, x_{1}\right)=\left(x_{0}, T x_{0}\right) \in E$. From (2.1), we have

$$
\tau+F\left(\operatorname{sp}\left(x_{1}, x_{2}\right)\right)=\tau+F\left(\operatorname{sp}\left(T x_{0}, T x_{1}\right)\right) \leq F\left(p\left(x_{0}, x_{1}\right)\right)
$$

As $T$ is edge preserving, for $\left(x_{0}, x_{1}\right) \in E$, we have $\left(x_{1}, x_{2}\right) \in E$, From (2.1), we have

$$
\tau+F\left(\operatorname{sp}\left(x_{2}, x_{3}\right)\right)=\tau+F\left(\operatorname{sp}\left(T x_{1}, T x_{2}\right)\right) \leq F\left(p\left(x_{1}, x_{2}\right)\right)
$$


Continuing in the same way, we get a sequence $\left\{x_{n}\right\} \subset X$ such that

$$
x_{n}=T x_{n-1}, x_{n-1} \neq x_{n} \text { and }\left(x_{n-1}, x_{n}\right) \in E \text { for each } n \in \mathbb{N} .
$$

Furthermore,

$$
\tau+F\left(\operatorname{sp}\left(x_{n}, x_{n+1}\right)\right) \leq F\left(p\left(x_{n-1}, x_{n}\right)\right) \text { for each } n \in \mathbb{N} .
$$

By using the property $F_{4}$, we have

$$
\tau+F\left(s^{n} p\left(x_{n}, x_{n+1}\right)\right) \leq F\left(s^{n-1} p\left(x_{n-1}, x_{n}\right)\right) .
$$

Letting $p_{n}=p\left(x_{n}, x_{n+1}\right)$, for each $n \in \mathbb{N}$ and after some simplification, we have

$$
F\left(s^{n} p_{n}\right) \leq F\left(p_{0}\right)-n \tau \text { for each } n \in \mathbb{N} \text {. }
$$

Letting $n \rightarrow \infty$ in (2.5), we get $\lim _{n \rightarrow \infty} F\left(s^{n} p_{n}\right)=-\infty$. Thus, by property $\left(F_{2}\right)$, we have $\lim _{n \rightarrow \infty} s^{n} p_{n}=0$. From $\left(F_{3}\right)$ there exists $k \in(0,1)$ such that

$$
\lim _{n \rightarrow \infty}\left(s^{n} p_{n}\right)^{k} F\left(s^{n} p_{n}\right)=0 \text {. }
$$

From (2.5) we have

$$
\left(s^{n} p_{n}\right)^{k} F\left(s^{n} p_{n}\right)-\left(s^{n} p_{n}\right)^{k} F\left(p_{0}\right) \leq-\left(s^{n} p_{n}\right)^{k} n \tau \leq 0 \text { for each } n \in \mathbb{N} .
$$

Letting $n \rightarrow \infty$ in (2.6), we get

$$
\lim _{n \rightarrow \infty} n\left(s^{n} p_{n}\right)^{k}=0 .
$$

This implies that there exists $n_{1} \in \mathbb{N}$ such that $n\left(s^{n} p_{n}\right)^{k} \leq 1$ for each $n \geq n_{1}$. Thus, we have

$$
s^{n} p_{n} \leq \frac{1}{n^{1 / k}}, \quad \text { for each } n \geq n_{1} .
$$

To show that $\left\{x_{n}\right\}$ is a $p$-Cauchy sequence, consider

$$
S_{n}=\sum_{i=n_{1}}^{n} \frac{1}{i^{1 / k}} .
$$

Since $\sum_{i=1}^{\infty} \frac{1}{i^{1 / k}}$ is convergent series. Thus, there exists $S \in[0, \infty)$ such that $\lim _{n \rightarrow \infty} S_{n}=S$. Consider $m, n \in \mathbb{N}$ with $m>n>n_{1}$. By using the triangular inequality and (2.8), we have

$$
\begin{aligned}
p\left(x_{n}, x_{m}\right) & \leq s^{n} p\left(x_{n}, x_{n+1}\right)+s^{n+1} p\left(x_{n+1}, x_{n+2}\right)+\cdots+s^{m-1} p\left(x_{m-1}, x_{m}\right) \\
& =\sum_{i=n_{1}}^{m-1} s^{i} p_{i}-\sum_{i=n_{1}}^{n-1} s^{i} p_{i} \\
& \leq S_{m-1}-S_{n-1} .
\end{aligned}
$$

Thus, $\lim _{n, m \rightarrow \infty} p\left(x_{n}, x_{m}\right)=0$. In a similar way, we show that $\lim _{n, m \rightarrow \infty} p\left(x_{m}, x_{n}\right)$ $=0$. Thus, $\left\{x_{n}\right\}$ is a $p$-Cauchy sequence. As $(X, \vartheta)$ is S-complete, there exists $x^{*} \in$ $X$ such that $\lim _{n \rightarrow \infty} p\left(x_{n}, x^{*}\right)=0$. By condition (iii), when $T$ is $p_{G}$-continuous, 
we have $\lim _{n \rightarrow \infty} p\left(x_{n+1}, T x^{*}\right)=0$. As $\lim _{n \rightarrow \infty} p\left(x_{n}, x^{*}\right)=0$ and $\lim _{n \rightarrow \infty} p\left(x_{n}, T x^{*}\right)=0$. Thus by Lemma 1-(a) we have $x^{*}=T x^{*}$. By condition (iii), when we have $\left(x_{n}, x^{*}\right) \in E$ for each $n \in \mathbb{N}$. From (2.1), we have

$$
\tau+F\left(\operatorname{sp}\left(T x_{n}, T x^{*}\right)\right) \leq F\left(p\left(x_{n}, x^{*}\right)\right) .
$$

This implies that $s p\left(x_{n+1}, T x^{*}\right)<p\left(x_{n}, x^{*}\right)$. Letting $n \rightarrow \infty$, we have $\lim _{n \rightarrow \infty} p\left(x_{n+1}, T x^{*}\right)=0$. Again, by Lemma 1-(a) we have $x^{*}=T x^{*}$.

Let $\Psi$ be the family of functions $\psi:[0, \infty) \rightarrow[0, \infty)$ satisfying the following conditions:

$\left(\Psi_{1}\right) \psi$ is nondecreasing;

$\left(\Psi_{2}\right) \sum_{n=1}^{+\infty} s^{n} \psi^{n}(t)<\infty$ for all $t>0$, where $\psi^{n}$ is the $n^{\text {th }}$ iterate of $\psi$.

Definition 7. Let $(X, \vartheta)$ be a uniform space endowed with the graph $G$ and $p$ is an $E_{S}$-distance on $X$. A mapping $T: X \rightarrow X$ is a $\psi_{G}$-contraction mapping if for each $(x, y) \in E$, we have

$$
p(T x, T y) \leq \psi(p(x, y))
$$

where $\psi \in \Psi$.

Theorem 2. Let $(X, \vartheta)$ be a $S$-complete Hausdorff uniform endowed with the graph $G$ and $p$ is an $E_{s}$-distance on $X$. Let $T: X \rightarrow X$ is a $\psi_{G}$-contraction mapping satisfying the following conditions:

(i) $T$ is edge preserving, that is, for $(x, y) \in E$, we have $(T x, T y) \in E$;

(ii) there exists $x_{0} \in X$ such that $\left(x_{0}, T x_{0}\right) \in E$ and $\left(T x_{0}, x_{0}\right) \in E$;

(iii) $T$ is $p_{G}$-continuous, or, for any sequence $\left\{x_{n}\right\} \subseteq X$ such that $x_{n} \rightarrow x$ as $n \rightarrow \infty$ and $\left(x_{n}, x_{n+1}\right) \in E$ for each $n \in \mathbb{N}$, we have $\left(x_{n}, x\right) \in E$ for each $n \in \mathbb{N}$.

\section{Then $T$ has a fixed point.}

Proof. By hypothesis (ii) of theorem we have $x_{0} \in X$ such that $\left(x_{0}, T x_{0}\right) \in E$. Define the sequence $\left\{x_{n}\right\}$ in $X$ by $x_{n+1}=T x_{n}$ for all $n \in \mathbb{N} \cup\{0\}$. If $x_{n_{0}}=x_{n_{0}+1}$ for some $n_{0}$, then $x_{n_{0}}$ is a fixed point of $T$. So, we can assume that $x_{n} \neq x_{n+1}$ for all $n$. Since $T$ is edge preserving, we have

$$
\left(x_{0}, x_{1}\right)=\left(x_{0}, T x_{0}\right) \in E \Rightarrow\left(T x_{0}, T x_{1}\right)=\left(x_{1}, x_{2}\right) \in E .
$$

Inductively, we have

$$
\left(x_{n}, x_{n+1}\right) \in E \text {, for all } n \in \mathbb{N} \cup\{0\} .
$$

From (2.9) and (2.10), it follows that for all $n \in \mathbb{N} \cup\{0\}$, we have

$$
p\left(x_{n+1}, x_{n+2}\right)=p\left(T x_{n}, T x_{n+1}\right) \leq \psi\left(p\left(x_{n}, x_{n+1}\right)\right) .
$$


Iteratively, we get

$$
p\left(x_{n}, x_{n+1}\right) \leq \psi^{n}\left(p\left(x_{0}, x_{1}\right)\right), \text { for all } n \in \mathbb{N} .
$$

Since $p$ is an $E_{s}$-distance then for $m>n$, we have

$$
\begin{aligned}
& p\left(x_{n}, x_{m}\right) \leq s^{n} p\left(x_{n}, x_{n+1}\right)+s^{n+1} p\left(x_{n+1}, x_{n+2}\right)+\cdots+s^{m-1} p\left(x_{m-1}, x_{m}\right) \\
& \leq s^{n} \psi^{n}\left(p\left(x_{0}, x_{1}\right)\right)+s^{n+1} \psi^{n+1}\left(p\left(x_{0}, x_{1}\right)\right)+\cdots+s^{m-1} \psi^{m-1}\left(p\left(x_{0}, x_{1}\right)\right) .
\end{aligned}
$$

To show that $\left\{x_{n}\right\}$ is a $p$-Cauchy sequence, consider

$$
S_{n}=\sum_{k=0}^{n} s^{k} \psi^{k}\left(p\left(x_{0}, x_{1}\right)\right) .
$$

Thus from (2) we have

$$
p\left(x_{n}, x_{m}\right) \leq S_{m-1}-S_{n-1} .
$$

Since $\psi \in \Psi$, there exists $S \in[0, \infty)$ such that $\lim _{n \rightarrow \infty} S_{n}=S$. Thus by (2.12) we have

$$
\lim _{n, m \rightarrow \infty} p\left(x_{n}, x_{m}\right)=0 .
$$

Since $p$ is not symmetric then by repeating the same argument we have $\lim _{n, m \rightarrow \infty} p\left(x_{m}, x_{n}\right)=0$. Hence the sequence $\left\{x_{n}\right\}$ is a $p$-Cauchy in the $S$-complete space $X$. Thus, there exists $x^{*} \in X$ such that $\lim _{n \rightarrow \infty} p\left(x_{n}, x^{*}\right)=0$. By condition (iii), when we have $T$ is $p_{G}$-continuous, we get $\lim _{n \rightarrow \infty} p\left(T x_{n}, T x^{*}\right)=0$ which implies that $\lim _{n \rightarrow \infty} p\left(x_{n+1}, T x^{*}\right)=0$. Hence we have $\lim _{n \rightarrow \infty} p\left(x_{n}, x^{*}\right)=0$ and $\lim _{n \rightarrow \infty}\left(x_{n}, T x^{*}\right)=0$. Thus by Lemma 1-(a) we have $x^{*}=T x^{*}$. By condition (iii), when we have $\left(x_{n}, x^{*}\right) \in E$ for each $n \in \mathbb{N}$, then from (2.9)

$$
p\left(x_{n+1}, T x^{*}\right)=p\left(T x_{n}, T x^{*}\right) \leq \psi\left(p\left(x_{n}, x^{*}\right)\right)<p\left(x_{n}, x^{*}\right) .
$$

Letting $n \rightarrow \infty$ in the above inequality, we have $\lim _{n \rightarrow \infty} p\left(x_{n+1}, T x^{*}\right)=0$. Thus by repeating the same arguments as above we have $x^{*}=T x^{*}$.

Example 3. Let $X=[0,1]$ be endowed with a graph $G=(V, E)$ with $V=X$ and $E=\left\{(x, y): x, y \in\left\{\frac{1}{n+1}: n \in \mathbb{N}\right\} \cup\{0\}\right\} \cup\{(x, x): x \in X\}$, and $b$-metric $d(x, y)=$ $(x-y)^{2}$ with $s=2$. Define $\vartheta=\left\{U_{\epsilon} \mid \epsilon>0\right\}$. It is easy to see that $(X, \vartheta)$ is a uniform space. Define $T: X \rightarrow X$ by

$$
T x=\left\{\begin{array}{l}
0 \text { if } x=0 \\
\frac{1}{3 n+1} \text { if } x=\frac{1}{n}: n>1 \\
\sqrt{x} \text { otherwise. }
\end{array}\right.
$$

Take $\psi(t)=\frac{t}{3}$ for all $t \geq 0$. It is easy to see that $T$ is edge preserving and $\psi_{G^{-}}$ contraction. Also for $x_{0}=\frac{1}{2}$ we have $\left(x_{0}, T x_{0}\right) \in E$ and $\left(T x_{0}, x_{0}\right) \in E$. Moreover for any sequence $\left\{x_{n}\right\}$ in $X$ with $x_{n} \rightarrow x$ as $n \rightarrow \infty$ and $\left(x_{n-1}, x_{n}\right) \in E$ for each $n \in \mathbb{N}$ we have $\left(x_{n}, x\right) \in E$ for each $n \in \mathbb{N}$. Therefore by Theorem 2, $T$ has a fixed point. 
To investigate the uniqueness of a fixed point, we consider the following condition:

$(H)$ : For all $x, y \in \operatorname{Fix}(T)$, there exists $z \in X$ such that $(z, x) \in E$ and $(z, y) \in$ E.

Here, $\operatorname{Fix}(T)$ denotes the set of all fixed points of $T$.

The following theorem guarantees the uniqueness of a fixed point.

Theorem 3. Adding the condition $(H)$ in the hypothesis of Theorem 2, we obtain the uniqueness of fixed point of $T$.

Proof. Suppose, on the contrary, that $u, v \in X$ are two distinct fixed points of $T$. From $(H)$, there exists $z \in X$ such that

$$
(z, u) \in E \text { and }(z, v) \in E .
$$

By using the fact that $T$ is edge preserving, from (2.16), we have

$$
\left(T^{n} z, u\right) \in E \text { and }\left(T^{n} z, v\right) \in E \text {, for all } n \in \mathbb{N} \cup\{0\} .
$$

We define the sequence $\left\{z_{n}\right\}$ in $X$ by $z_{n+1}=T z_{n}=T^{n} z_{0}$ for all $n \in \mathbb{N} \cup\{0\}$ and $z_{0}=z$. From (2.17) and (2.9), we have

$$
p\left(z_{n+1}, u\right)=p\left(T z_{n}, T u\right) \leq \psi\left(p\left(z_{n}, u\right)\right),
$$

for all $n \in \mathbb{N} \cup\{0\}$. This implies that

$$
p\left(z_{n}, u\right) \leq \psi^{n}\left(p\left(z_{0}, u\right)\right) \text {, for all } n \in \mathbb{N} .
$$

Letting $n \rightarrow \infty$ in the above inequality, we obtain

$$
\lim _{n \rightarrow \infty} p\left(z_{n}, u\right)=0 .
$$

Similarly, we have

$$
\lim _{n \rightarrow \infty} p\left(z_{n}, v\right)=0 .
$$

From (2.19) and (2.20) together with Lemma 1-(a), it follows that $u=v$. Thus, fixed point of $T$ is unique.

Definition 8. Let $(X, \vartheta)$ be a uniform space endowed with the graph $G$. A pair of two self mappings $T, S: X \rightarrow X$ is said to be a $\psi_{G}$-contraction pair if for each $(x, y) \in E$, we have

$$
\max \{p(T x, S y), p(S x, T y)\} \leq \psi(p(x, y)),
$$

where $\psi \in \Psi$.

Theorem 4. Let $(X, \vartheta)$ be a $S$-complete Hausdorff uniform space endowed with the graph $G$ and $p$ is an $E_{S}$-distance on $X$. Suppose that the pair of $T, S: X \rightarrow X$ is $\psi_{G}$-contraction pair satisfying the following conditions.

(i) $(T, S)$ is edge preserving pair, that is, for each $(x, y) \in E$, we have $(T x, S y) \in$ $E$ and $(S x, T y) \in E$;

(ii) there exists $x_{0} \in X$ such that $\left(x_{0}, T x_{0}\right) \in E$ and $\left(T x_{0}, x_{0}\right) \in E$; 
(iii) for any sequence $\left\{x_{n}\right\}$ in $X$ with $x_{n} \rightarrow x$ as $n \rightarrow \infty$ and $\left(x_{n}, x_{n+1}\right) \in E$ for each $n \in \mathbb{N} \cup\{0\}$, then $\left(x_{n}, x\right) \in E$ for each $n \in \mathbb{N} \cup\{0\}$.

Then $T$ and $S$ have a common fixed point.

Proof. By hypothesis (ii) of theorem, we have $x_{0} \in X$ such that $\left(x_{0}, T x_{0}\right) \in E$ and $\left(T x_{0}, x_{0}\right) \in E$. Since $(T, S)$ is an edge preserving pair, then we can construct a sequence such that

$T x_{2 n}=x_{2 n+1}, S x_{2 n+1}=x_{2 n+2}$ and $\left(x_{n}, x_{n+1}\right) \in E,\left(x_{n+1}, x_{n}\right) \in E, \mathbb{N} \cup\{0\}$, for all $n \in \mathbb{N} \cup\{0\}$. From (2.21) for all $n \in \mathbb{N} \cup\{0\}$, we have

$$
\begin{aligned}
p\left(x_{2 n+1}, x_{2 n+2}\right) & =p\left(T x_{2 n}, S x_{2 n+1}\right) \\
& \leq \max \left\{p\left(T x_{2 n}, S x_{2 n+1}\right), p\left(S x_{2 n}, T x_{2 n+1}\right)\right\} \\
& \leq \psi\left(p\left(x_{2 n}, x_{2 n+1}\right)\right) .
\end{aligned}
$$

Hence, we conclude that

$$
p\left(x_{2 n+1}, x_{2 n+2}\right) \leq \psi\left(p\left(x_{2 n}, x_{2 n+1}\right)\right) .
$$

Similarly, we get

$$
\begin{aligned}
p\left(x_{2 n+2}, x_{2 n+3}\right) & =p\left(S x_{2 n+1}, T x_{2 n+2}\right) \\
& \leq \max \left\{p\left(T x_{2 n+1}, S x_{2 n+2}\right), p\left(S x_{2 n+1}, T x_{2 n+2}\right)\right\} \\
& \leq \psi\left(p\left(x_{2 n+1}, x_{2 n+2}\right)\right) .
\end{aligned}
$$

Hence, we have

$$
p\left(x_{2 n+2}, x_{2 n+3}\right) \leq \psi\left(p\left(x_{2 n+1}, x_{2 n+2}\right)\right) .
$$

Thus from (2.22) and (2.23), and by induction, we get

$$
p\left(x_{n}, x_{n+1}\right) \leq \psi^{n}\left(p\left(x_{0}, x_{1}\right)\right), \text { for all } n \in \mathbb{N} \text {. }
$$

Now we show that $\left\{x_{n}\right\}$ is a $p$-Cauchy sequence. Since $p$ is an $E_{s}$-distance then for $m>n$, we have

$$
\begin{aligned}
& p\left(x_{n}, x_{m}\right) \leq s^{n} p\left(x_{n}, x_{n+1}\right)+s^{n+1} p\left(x_{n+1}, x_{n+2}\right)+\cdots+s^{m-1} p\left(x_{m-1}, x_{m}\right) \\
& \leq s^{n} \psi^{n}\left(p\left(x_{0}, x_{1}\right)\right)+s^{n+1} \psi^{n+1}\left(p\left(x_{0}, x_{1}\right)\right)+\cdots+s^{m-1} \psi^{m-1}\left(p\left(x_{0}, x_{1}\right)\right) .
\end{aligned}
$$

Now, we shall consider

$$
S_{n}=\sum_{k=0}^{n} s^{k} \psi^{k}\left(p\left(x_{0}, x_{1}\right)\right) .
$$

Thus, from (2) we have

$$
p\left(x_{n}, x_{m}\right) \leq S_{m-1}-S_{n-1} .
$$

Since $\psi \in \Psi$, there exists $S \in[0, \infty)$ such that $\lim _{n \rightarrow \infty} S_{n}=S$. Thus, by (2.25) we have

$$
\lim _{n, m \rightarrow \infty} p\left(x_{n}, x_{m}\right)=0 .
$$


Since $p$ is not symmetric then by repeating the same argument we have

$$
\lim _{n, m \rightarrow \infty} p\left(x_{m}, x_{n}\right)=0 .
$$

Hence the sequence $\left\{x_{n}\right\}$ is a $p$-Cauchy in the $S$-complete space $X$. Thus, there exists $x^{*} \in X$ such that $\lim _{n \rightarrow \infty} p\left(x_{n}, x^{*}\right)=0$ which implies $\lim _{n \rightarrow \infty} T x_{2 n}=$ $\lim _{n \rightarrow \infty} S x_{2 n+1}=x^{*}$. By assumption (iii), we have $\left(x_{n}, x^{*}\right) \in E$. Thus, by using the triangular inequality and (2.21), we have

$$
\begin{aligned}
p\left(x_{n}, T x^{*}\right) & \leq s p\left(x_{n}, x_{2 n+2}\right)+s p\left(x_{2 n+2}, T x^{*}\right) \\
& =s p\left(x_{n}, x_{2 n+2}\right)+s p\left(S x_{2 n+1}, T x^{*}\right) \\
& \leq s p\left(x_{n}, x_{2 n+2}\right)+s \max \left\{p\left(T x_{2 n+1}, S x^{*}\right), p\left(S x_{2 n+1}, T x^{*}\right)\right\} \\
& \leq s p\left(x_{n}, x_{2 n+2}\right)+s \psi\left(p\left(x_{2 n+1}, x^{*}\right)\right)
\end{aligned}
$$

Letting $n \rightarrow \infty$ in (2.28), we have $p\left(x_{n}, T x^{*}\right)=0$. Hence we have $\lim _{n \rightarrow \infty} p\left(x_{n}, x^{*}\right)=0$ and $\lim _{n \rightarrow \infty} p\left(x_{n}, T x^{*}\right)=0$. Thus by Lemma 1-(a) we have $x^{*}=T x^{*}$. Analogously, we can drive $x^{*}=S x^{*}$. Therefore $x^{*}=T x^{*}=S x^{*}$.

Remark 2. Note that Theorem 4 is valid if one replace condition (ii) with

(ii)': there exists $x_{0} \in X$ such that $\left(x_{0}, S x_{0}\right) \in E$ and $\left(S x_{0}, x_{0}\right) \in E$.

Example 4. Let $X=[0,1]$ be endowed with a graph $G=(V, E)$ with $V=X$ and $E=\left\{(x, y): x, y \in\left\{\frac{1}{n+1}: n \in \mathbb{N}\right\} \cup\{0\}\right\} \cup\{(x, x): x \in X\}$, and dislocated metric space $d(x, y)=\max \{x, y\}$. Define $\vartheta=\left\{U_{\epsilon} \mid \epsilon>0\right\}$, where $U_{\epsilon}=\left\{(x, y) \in X^{2}\right.$ : $d(x, y)<d(x, x)+\epsilon\}$. It is easy to see that $(X, \vartheta)$ is a uniform space. Define $T: X \rightarrow X$ by

$$
T x=\left\{\begin{array}{l}
0 \text { if } x=0 \\
\frac{1}{2 n+1} \text { if } x=\frac{1}{n}: n>1 \\
x^{2} \text { otherwise }
\end{array}\right.
$$

and $S: X \rightarrow X$ by

$$
S x=\left\{\begin{array}{l}
0 \text { if } x=0 \\
\frac{1}{2 n} \text { if } x=\frac{1}{n}: n>1 \\
\sqrt{x} \text { otherwise }
\end{array}\right.
$$

Take $\psi(t)=\frac{t}{2}$ for all $t \geq 0$. Further, it is easy to see that $(T, S)$ is edge preserving and $\psi_{G}$-contraction pair. Also for $x_{0}=\frac{1}{2}$ we have $\left(x_{0}, T x_{0}\right) \in E$ and $\left(T x_{0}, x_{0}\right) \in E$. Moreover for any sequence $\left\{x_{n}\right\}$ in $X$ with $x_{n} \rightarrow x$ as $n \rightarrow \infty$ and $\left(x_{n}, x_{n+1}\right) \in E$ for each $n \in \mathbb{N} \cup\{0\}$ we have $\left(x_{n}, x\right) \in E$ for each $n \in \mathbb{N}$. Therefore by Theorem 4, $T$ and $S$ have a common fixed point.

To investigate the uniqueness of a common fixed point, we use the following condition.

(I) For each $x, y \in C F i x(T, S)$, we have $(x, y) \in E$, where $C F i x(T, S)$ is the set of all common fixed points of $T$ and $S$. 
Theorem 5. Adding the condition (I) in the hypothesis of Theorem 4, we obtain the uniqueness of common fixed point of $T$ and $S$.

Proof. On the contrary suppose that $u, v \in X$ are two distinct common fixed points of $T$ and $S$. From $(I)$ and (2.21) we have

$$
p(u, v)=\max \{p(T u, S v), p(S u, T v)\} \leq \psi(p(u, v))<p(u, v),
$$

which is impossible for $p(u, v)>0$. Consequently, we have $p(u, v)=0$. Analogously, one can show that $p(u, u)=0$. Thus we have $u=v$, which is a contradiction to our assumption. Hence $T$ and $S$ have a unique common fixed point.

\section{ACKNOWLEDGEMENT}

The authors declare that there is no conflict of interests regarding the publication of this article. All authors have equally contributed in writing this article. All authors have read and approved the final manuscript.

\section{REFERENCES}

[1] M. Aamri, S. Bennani, and D. El Moutawakil, "Fixed points and variational principle in uniform spaces," Sib. Ėlektron. Mat. Izv., vol. 3, pp. 137-142 (electronic), 2006.

[2] M. Aamri and D. El Moutawakil, "Common fixed point theorems for $E$-contractive or $E$ expansive maps in uniform spaces," Acta Math. Acad. Paedagog. Nyházi. (N.S.), vol. 20, no. 1, pp. 83-91 (electronic), 2004.

[3] M. Aamri and D. El Moutawakil, "Weak compatibility and common fixed point theorems for A-contractive and E-expansive maps in uniform spaces," Serdica Math. J., vol. 31, no. 1-2, pp. 75-86, 2005.

[4] R. P. Agarwal, D. O'Regan, and N. S. Papageorgiou, "Common fixed point theory for multivalued contractive maps of Reich type in uniform spaces," Appl. Anal., vol. 83, no. 1, pp. 37-47, 2004, doi: 10.1080/00036810310001620063. [Online]. Available: http://dx.doi.org/10.1080/00036810310001620063

[5] F. Bojor, "Fixed point of $\phi$-contraction in metric spaces endowed with a graph," An. Univ. Craiova Ser. Mat. Inform., vol. 37, no. 4, pp. 85-92, 2010.

[6] F. Bojor, "Fixed point theorems for Reich type contractions on metric spaces with a graph," Nonlinear Anal., vol. 75, no. 9, pp. 3895-3901, 2012, doi: 10.1016/j.na.2012.02.009. [Online]. Available: http://dx.doi.org/10.1016/j.na.2012.02.009

[7] M. Cosentino, M. Jleli, B. Samet, and C. Vetro, "Solvability of integrodifferential problems via fixed point theory in $b$-metric spaces," Fixed Point Theory Appl., pp. 2015:70, 15, 2015, doi: 10.1186/s13663-015-0317-2. [Online]. Available: http://dx.doi.org/10.1186/s13663-015-0317-2

[8] M. Cosentino and P. Vetro, "Fixed point results for $F$-contractive mappings of Hardy-Rogerstype," Filomat, vol. 28, no. 4, pp. 715-722, 2014, doi: 10.2298/FIL1404715C. [Online]. Available: http://dx.doi.org/10.2298/FIL1404715C

[9] S. Czerwik, "Contraction mappings in b-metric spaces," Acta Math. Inform. Univ. Ostraviensis, vol. 1, pp. 5-11, 1993.

[10] J. Jachymski, "The contraction principle for mappings on a metric space with a graph," Proc. Amer. Math. Soc., vol. 136, no. 4, pp. 1359-1373, 2008, doi: 10.1090/S0002-9939-07-09110-1. [Online]. Available: http://dx.doi.org/10.1090/S0002-9939-07-09110-1 
[11] T. Kamran, M. Samreen, and N. Shahzad, "Probabilistic G-contractions," Fixed Point Theory Appl., pp. 2013:223, 14, 2013, doi: 10.1186/1687-1812-2013-223. [Online]. Available: http://dx.doi.org/10.1186/1687-1812-2013-223

[12] A. Nicolae, D. O'Regan, and A. Petruşel, "Fixed point theorems for singlevalued and multivalued generalized contractions in metric spaces endowed with a graph," Georgian Math. J., vol. 18, no. 2, pp. 307-327, 2011.

[13] D. Paesano and C. Vetro, "Multi-valued $F$-contractions in 0-complete partial metric spaces with application to Volterra type integral equation," Rev. R. Acad. Cienc. Exactas Fís. Nat. Ser. A Math. RACSAM, vol. 108, no. 2, pp. 1005-1020, 2014, doi: 10.1007/s13398-013-0157-z. [Online]. Available: http://dx.doi.org/10.1007/s13398-013-0157-z

[14] M. Samreen, T. Kamran, and N. Shahzad, "Some fixed point theorems in $b$-metric space endowed with graph," Abstr. Appl. Anal., pp. Art. ID 967 132, 9, 2013.

[15] N.-A. Secelean, "Iterated function systems consisting of F-contractions," Fixed Point Theory Appl., pp. 2013:277, 13, 2013, doi: 10.1186/1687-1812-2013-277. [Online]. Available: http://dx.doi.org/10.1186/1687-1812-2013-277

[16] M. Sgroi and C. Vetro, "Multi-valued $F$-contractions and the solution of certain functional and integral equations," Filomat, vol. 27, no. 7, pp. 1259-1268, 2013, doi: 10.2298/FIL1307259S. [Online]. Available: http://dx.doi.org/10.2298/FIL1307259S

[17] J. Tiammee and S. Suantai, "Coincidence point theorems for graph-preserving multi-valued mappings," Fixed Point Theory Appl., pp. 2014:70, 11, 2014, doi: 10.1186/1687-1812-2014-70. [Online]. Available: http://dx.doi.org/10.1186/1687-1812-2014-70

[18] C. Vetro and F. Vetro, "Metric or partial metric spaces endowed with a finite number of graphs: a tool to obtain fixed point results," Topology Appl., vol. 164, pp. 125-137, 2014, doi: 10.1016/j.topol.2013.12.008. [Online]. Available: http://dx.doi.org/10.1016/j.topol.2013.12.008

[19] D. Wardowski, "Fixed points of a new type of contractive mappings in complete metric spaces," Fixed Point Theory Appl., pp. 2012:94, 6, 2012, doi: 10.1186/1687-1812-2012-94. [Online]. Available: http://dx.doi.org/10.1186/1687-1812-2012-94

[20] E. Zeidler, Nonlinear functional analysis and its applications. I Fixed-point theorems. SpringerVerlag, New York, 1986, translated from the German by Peter R. Wadsack. [Online]. Available: http://dx.doi.org/10.1007/BF00047050. doi: 10.1007/BF00047050

Authors' addresses

Muhammad Usman Ali

Department of Sciences and Humanities, National University of Computer and Emerging Sciences (FAST), Islamabad-Pakistan

E-mail address: muh_usman_ali@yahoo.com

\section{Fahimuddin}

Department of Mathematics, Quaid-i-Azam University, Islamabad-Pakistan and Center for Advanced Studies in Engineering (CASE), Islamabad-Pakistan

E-mail address: fahamiludgmail.com

\section{Tayyab Kamran}

Department of Mathematics, Quaid-i-Azam University, Islamabad-Pakistan

E-mail address: tayyabkamranegmail.com 


\section{Erdal Karapinar}

Department of Mathematics, Atilim University 06836, Incek, Ankara-Turkey, and Nonlinear Analysis and Applied Mathematics (NAAM) Research Group, King Abdulaziz University, 21589, Jeddah , Saudi Arabia

E-mail address: erdalkarapinar@yahoo.com 